\title{
Predação por coccinelídeos e crisopídeo influenciada pela teia de Tetranychus evansi
}

\author{
Madelaine Venzon ${ }^{(1)}$, Felipe Lemos(2), Renato Almeida Sarmento(3), Maria Consolação Rosado(2) e Angelo Pallini(2) \\ (1)Empresa de Pesquisa Agropecuária de Minas Gerais, Unidade Regional Epamig Zona da Mata, Vila Gianetti, ํo 46, CEP 36570-000 Viçosa, \\ MG. E-mail: venzon@epamig.ufv.br (2)Universidade Federal de Viçosa, Departamento de Biologia Animal, Avenida P.H. Rolfs, s/no, Campus \\ UFV, CEP 36570-000 Viçosa, MG. E-mail: felipelemosufv@yahoo.com.br, mcrmartins@yahoo.com.br, pallini@ufv.br (3)Universidade Federal \\ do Tocantins, Campus de Gurupi, Rua Badejós, Chácaras 69 e 72, Lote 07, Zona Rural, CEP 77404-970 Gurupi. E-mail: rsarmento@uft.edu.br
}

Resumo - O objetivo deste trabalho foi avaliar o efeito da teia produzida por Tetranychus evansi (Acari: Tetranychidae) na eficiência de predação por coccinelídeos Cycloneda sanguinea e Eriopis connexa e pelo crisopídeo Chrysoperla externa. A eficiência em predar as fêmeas de T. evansi na presença e na ausência de sua teia em discos de folhas de tomateiro com ácaros foi avaliada para cada instar das diferentes espécies de predadores. Foi observado efeito negativo da teia produzida por $T$. evansi na eficiência de predação do segundo e do terceiro instar de C. externa. Quanto à E. connexa, somente a predação pelo último instar foi influenciada negativamente pela presença da teia. A predação pelas larvas de primeiro e terceiro instar de C. sanguinea foi significativamente menor na presença da teia; no entanto, o resultado foi contrário para o quarto instar. O efeito da teia de T. evansi como mecanismo de defesa varia conforme a espécie e o estágio de desenvolvimento do predador.

Termos para indexação: Chrysoperla externa, Cycloneda sanguinea, Eriopis connexa, ácaro-vermelho do tomateiro, mecanismo de defesa.

\section{Predation by coccinellids and chrysopid species influenced by Tetranychus evansi webbing}

\begin{abstract}
The aim of this work was to evaluate the effect of the webbing produced by Tetranychus evansi (Acari: Tetranychidae) on the predation efficiency of Cycloneda sanguinea and Eriopis connexa coccinellids and of the Chrysoperla externa chrysopid. The predation efficiency on T. evansi females with and without webbing on tomato leaf discs was evaluated for each juvenile stage of the different predator species tested. There was a negative effect of T. evansi webbing on the predation efficiency of the second and third C. externa juvenile stages. Regarding E. connexa, only the predation by the oldest juvenile stage was negatively affected by the webbing. The predation efficiency of the first and the third juvenile stages of $C$. sanguinea was lower in the presence of webbing, but the opposite was observed for the fourth stage. The defensive role of $T$. evansi webbing varies according to the predator species and their developmental stage.
\end{abstract}

Index terms: Chrysoperla externa, Cycloneda sanguinea, Eriopis connexa, red tomato spider mite, defense mechanisms.

\section{Introdução}

Interações entre artrópodes fitófagos e seus inimigos naturais podem ser influenciadas pelas características inerentes tanto às espécies de inimigos naturais quanto à planta hospedeira. A eficiência de predação pode ser reduzida ou aumentada de acordo com a complexidade à superfície da planta hospedeira. A presença de tricomas nas plantas pode causar repelência aos herbívoros e aos seus inimigos naturais, e também pode impedir os movimentos desses artrópodes e/ou aumentar a área a ser forrageada pelos predadores (Gamarra et al., 1998; Krips et al., 1999; Roda et al., 2000; Michalska, 2003). A existência de locais de refúgio para as presas, como as domácias foliares, também pode reduzir a taxa de predação (Roda et al., 2000, 2001; Matos et al., 2006). Além disso, estruturas produzidas pelos artrópodes na superfície das folhas, como as teias dos ácaros da família Tetranychidae, podem dificultar o deslocamento de alguns microartrópodos (Sabelis \& Bakker, 1992). Essas teias podem interferirnegativamentena habilidade predatória de algumas espécies de ácaros (Cloutier \& 
Johnson, 1993; Pallini et al., 1998; Vantornhout et al., 2005) e de insetos predadores (Venzon et al., 2000).

Oácaro Tetranychus evansi Baker \& Pritchard (Acari: Tetranychidae) gasta muito tempo para se alimentar do parênquima foliar e, por ser lento, torna-se uma vítima fácil para predadores mais ágeis (Saito, 1985). Para se defender dos seus inimigos naturais, T. evansi produz uma grande quantidade de teia sobre suas colônias, o que dificulta a ação dos predadores (Gerson, 1985; Sabelis \& Bakker, 1992). Algumas espécies de ácaros predadores, no entanto, podem sobrepor essas defesas por meio de especializações ou adaptações morfológicas de suas setas dorsais, que os ajudam a reduzir o contato do corpo com os fios da teia produzida por T. evansi e, dessa forma, reduzem as chances de ficarem presas na teia e morrerem de fome (Sabelis \& Bakker, 1992; Sabelis et al., 1994). Contudo, mesmo aqueles ácaros predadores mais adaptados têm dificuldade de penetrar na teia de T. evansi (Moraes \& McMurtry, 1985; Rosa et al., 2005). Além disso, a inadequação nutricional de T. evansi a vários ácaros predadores tem dificultado a seleção de agentes de controle biológico para esse ácaro fitófago, pois alguns ácaros predadores apresentaram baixa taxa de oviposição e redução da sobrevivência quando alimentados com $T$. evansi (Moraes \& McMurtry, 1985; Rosa et al., 2005). Uma exceção é a espécie Phytoseiulus longipes Evans, que tem demonstrado desempenho satisfatório quando alimentada com T. evansi (Ferrero et al., 2007; Furtado et al., 2007).

O ácaro T. evansi ocorre em plantas da família Solanaceae, principalmente em cultivos de tomate (Flechtmann, 1983; Bonato, 1999; Sarmento et al., 2004; Wekesa et al., 2005; Oliveira et al., 2005). Várias espécies de predadores, além de ácaros, ocorrem em cultivos de tomate, com destaque para os coccinelídeos Cycloneda sanguinea L. e Eriopis connexa Mulsant (Coleoptera: Coccinellidae) e o crisopídeo Chrysoperla externa (Hagen) (Neuroptera: Chrysopidae), os quais predam pulgões, ovos, larvas e insetos de corpo mole e ácaros (Toscano et al., 2003; Bettiol et al., 2004; Sarmento et al., 2004; Oliveira et al., 2005; Picanço et al., 2007). Apesar dos relatos de que essas espécies predam ácaros, pouco se sabe sobre a eficiência delas em reduzir as populações de tetraniquídeos, em especial de T. evansi. Com base nos estudos desenvolvidos com ácaros predadores, a inadequação nutricional e a presença da teia densa de T. evansi são fatores que influenciam negativamente o desempenho de algumas espécies de predadores em controlar essa espécie (Moraes \& McMurtry, 1985; Rosa et al., 2005; Furtado et al., 2007).

O objetivo deste trabalho foi avaliar o efeito da teia produzida por Tetranychus evansi (Acari: Tetranychidae) na eficiência de predação dos coccinelídeos C. sanguinea e E. connexa e do crisopídeo C. externa.

\section{Material e Métodos}

Adultos de C. sanguinea e E. connexa foram coletados em plantas de tomate de uma área experimental localizada no campus da Universidade Federal de Viçosa (UFV) e transferidos para laboratório $\left(25 \pm 2^{\circ} \mathrm{C}\right.$, $70 \pm 10 \%$ UR e 14 horas de fotófase). Para a criação dos predadores, cinco casais de cada espécie foram acondicionados em copos plásticos $(500 \mathrm{~mL})$ cuja tampa apresentava uma abertura central $(\varnothing=2 \mathrm{~cm})$ revestida com organza. A alimentação consistiu de pulgões e ácaros de diversas espécies, fornecidos em folhas de tomate. Os ovos depositados pelas fêmeas no interior dos copos plásticos foram recolhidos com o auxílio de um pincel e transferidos para placas de Petri $(5 \times 1,5 \mathrm{~cm})$ que continham um chumaço de algodão umedecido, onde foram mantidos até a eclosão das larvas. Estas foram utilizadas para os experimentos ou para a manutenção da criação e foram alimentadas da mesma forma que os adultos.

As larvas de C. externa foram obtidas de uma criação mantida no Laboratório de Entomologia $\left(25 \pm 2^{\circ} \mathrm{C}\right.$, $70 \pm 10 \%$ UR e 14 horas de fotófase) da Empresa de Pesquisa Agropecuária de Minas Gerais (Epamig), em Viçosa, MG. Essa criação foi estabelecida com insetos oriundos do Laboratório de Entomologia da Universidade Federal de Lavras (Ufla), Lavras, MG, em 2004 e foi periodicamente revigorada com a introdução de insetos coletados em áreas experimentais da Epamig e de insetos oriundos do Município de Lavras. Os adultos foram mantidos no laboratório, em gaiolas de PVC $(15 \times 15 \mathrm{~cm})$ revestidas com papel toalha branco. As gaiolas foram fechadas com filme de PVC nas extremidades superiores, e as extremidades inferiores foram apoiadas em placa de Petri forrada com papel toalha branco. A alimentação consistiu de uma mistura de levedo de cerveja e mel (1:1) pincelada em tira de Parafilm (Pechiney Plastic Packaging Company, Chicago, EUA) presa internamente na parte superior da gaiola. As tiras que continham a alimentação foram

Pesq. agropec. bras., Brasília, v.44, n.9, p.1086-1091, set. 2009 
substituídas a cada dois dias. Também foi fornecida água em um frasco de $10 \mathrm{~mL}$ com um chumaço de algodão. Os ovos foram individualizados em tubos plásticos de $40 \mathrm{~mL}$ vedados com filme de PVC na extremidade superior. A alimentação das larvas consistiu de pulgões - Myzus persicae (Sulzer, 1776) e Toxoptera citricida (Kirkaldy, 1907) - e de ácaros - Tetranychus urticae, T. evansi-, fornecidos a cada três dias.

$\mathrm{Na}$ realização dos experimentos, foram utilizadas arenas constituídas de placas de Petri $(\varnothing=3,5 \mathrm{~cm}) \mathrm{com}$ $1 \mathrm{~cm}$ de altura. No fundo de cada placa, foi colocado um algodão umedecido e, em seguida, foi acondicionado um disco $(\varnothing=3 \mathrm{~cm})$ feito a partir de folhas de plantas de tomate. Os discos foram posicionados com a face abaxial da folha voltada para cima. Para testar o efeito da teia sobre a eficiência de predação dos insetos, 20 fêmeas de T. evansi foram transferidas para cada arena para testar a predação pelo primeiro e segundo instares dos predadores; 40 fêmeas de $T$. evansi, para testar a predação do terceiro instar, e 70 fêmeas, para avaliar a predação do quarto instar dos coccinelídeos.

As fêmeas dos ácaros foram deixadas nas arenas por dois dias, para se alimentar e produzir teia. No experimento sem a presença da teia, as larvas dos predadores foram adicionadas às arenas $20 \mathrm{~min}$ após a transferência dos ácaros. As larvas, colocadas individualmente em cada disco, estavam sem alimentação há 12 horas.

A influência da teia na predação em cada espécie de predador foi avaliada em experimentos distintos. O delineamento experimental adotado foi o inteiramente casualizado, e os tratamentos foram dispostos em esquema fatorial (instar $\mathrm{x}$ teia). Para $C$. sanguinea e E. connexa, o experimento foi composto por oito tratamentos, representados pelos quatro instares e pela presença ou ausência de teia. Para C. externa, o experimento foi composto por seis tratamentos, representados pelos três instares e pela presença ou ausência de teia. Cada tratamento foi repetido dez vezes, e a parcela experimental foi representada por uma arena com um disco de folha com T. evansi e uma larva de cada predador.

O número de ácaros mortos em cada disco foi avaliado 24 horas após a introdução das larvas dos predadores. Após a avaliação, as larvas dos predadores foram transferidas para novas arenas previamente preparadas, em intervalos iguais aos utilizados anteriormente (dois dias para T. evansi com teia e $20 \mathrm{~min}$ para T. evansi sem teia). Uma segunda avaliação foi feita após 24 horas. Os dados que representam a proporção de ácaros mortos em relação ao total de ácaros oferecidos nos dois dias foram analisados por meio de modelo linear generalizado (GLM), e erros do tipo quasibinomial com link logit foram analisados com o software RGui 2.7 (Crawley, 2007). Modelos com interação entre dois fatores (teia $\mathrm{x}$ instar) para cada uma das espécies de predadores foram utilizados na análise. A interação foi excluída do modelo quando não significativa a $5 \%$ de probabilidade. Posteriormente, os modelos foram submetidos à ANOVA com o teste $\mathrm{F}$, e as diferenças entre as médias foram obtidas por meio do teste de Wald fornecido pelo pacote "contrast" (Kuhn et al., 2008).

\section{Resultados e Discussão}

Verificou-se interação do efeito da teia de T. evansi com os instares do predador E. connexa em relação à percentagem de ácaros mortos $\left(\mathrm{F}_{3,62}=3,50 ; \mathrm{p}<0,05\right)$. A presença da teia não interferiu na predação pelas larvas do primeiro ao terceiro instar. No entanto, para o quarto instar, a percentagem de ácaros mortos pelo predador foi reduzida na presença da teia $(\mathrm{g} .1 .=62$, $\mathrm{t}=2,98, \mathrm{p}<0,01)($ Tabela 1).

Tabela 1. Percentagem média \pm erro padrão de ácaros Tetranychus evansi predados pelos diferentes instares larvais de duas espécies de coccinelídeos e uma de crisopídeo em discos foliares de plantas de tomate na presença e na ausência de teia do ácaro ${ }^{(1)}$.

\begin{tabular}{|c|c|c|c|c|c|c|}
\hline \multirow[t]{2}{*}{ Predador } & \multirow[t]{2}{*}{ Tratamento } & \multicolumn{5}{|c|}{ Predação (\%) } \\
\hline & & $1^{\mathrm{o}}$ instar & $2^{\circ}$ instar & $3^{\circ}$ instar & $4^{\mathrm{O}}$ instar & $\mathrm{CV}(\%)$ \\
\hline \multirow{2}{*}{ Eriopis connexa } & Com teia & $44,17 \pm 7,30 \mathrm{~A}$ & $95, \overline{56 \pm 2,79 \mathrm{~A}}$ & $94,25 \pm 2,84 \mathrm{~A}$ & $73,67 \pm 13,02 \mathrm{~A}$ & \multirow[b]{2}{*}{35,68} \\
\hline & Sem teia & $43,61 \pm 9,23 \mathrm{~A}$ & $85,31 \pm 6,40 \mathrm{~A}$ & $99,88 \pm 0,13 \mathrm{~A}$ & $95,63 \pm 3,19 \mathrm{~B}$ & \\
\hline \multirow{2}{*}{ Cycloneda sanguinea } & Com teia & $29,50 \pm 3,45 \mathrm{~A}$ & $41,25 \pm 3,23 \mathrm{~A}$ & $36,67 \pm 2,43 \mathrm{~A}$ & $55,27 \pm 6,22 \mathrm{~A}$ & \multirow{2}{*}{41,28} \\
\hline & Sem teia & $55,00 \pm 5,32 \mathrm{~B}$ & $32,50 \pm 3,62 \mathrm{~A}$ & $69,86 \pm 6,91 \mathrm{~B}$ & $39,86 \pm 5,80 \mathrm{~B}$ & \\
\hline \multirow{2}{*}{ Chrysoperla externa } & Com teia & $33,33 \pm 6,73 \mathrm{~A}$ & $46,25 \pm 11,23 \mathrm{~A}$ & $37,29 \pm 8,54 \mathrm{~A}$ & - & \multirow[b]{2}{*}{49,26} \\
\hline & Sem teia & $55,50 \pm 6,64 \mathrm{~A}$ & $90,00 \pm 5,34 \mathrm{~B}$ & $63,63 \pm 9,15 \mathrm{~B}$ & - & \\
\hline
\end{tabular}

${ }^{(1)}$ Médias seguidas por letras iguais na coluna para cada predador não diferem entre si pelo teste de Wald, a 5\% de probabilidade. 
A presença da teia de $T$. evansi afetou também os instares larvais de $C$. sanguinea quanto à percentagem de ácaros predados por esse coccinelídeo $\left(F_{3,56}=11,60 ; p<0,001\right)$. Larvas de primeiro e terceiro instar foram negativamente afetadas pela presença da teia (g.l. $=56, \mathrm{t}=2,46, \mathrm{p}<0,05$ e g.l. $=56, \mathrm{t}=4,83$, $\mathrm{p}<0,001$, respectivamente). Contudo, não houve efeito da teia na eficiência de predação das larvas do segundo instar (g.l. $=56, \mathrm{t}=0,9, \mathrm{p}=0,37)$ e, no último instar, a predação foi maior em discos foliares com teia (g.1. $=56, \mathrm{t}=2,49, \mathrm{p}<0,05)$ (Tabela 1).

Quanto ao predador C. externa, a percentagem de ácaros predados foi influenciada pela presença da teia de $T$. evansi $\left(\mathrm{F}_{1,46}=15,69 ; \mathrm{p}<0,001\right)$. No entanto, não houve interação significativa entre os instares larvais e a presença ou a ausência de teia $\left(\mathrm{F}_{2,42}=1,25\right.$, $\mathrm{p}=0,297)$. Não houve diferença significativa, para o primeiro instar, entre a percentagem de ácaros predados na presença e na ausência de teia (g.l. $=42$, $\mathrm{t}=1,42, \mathrm{p}>0,05)$. Quanto às larvas de segundo $\mathrm{e}$ terceiro instares, a predação de T. evansi foi menor nos discos com teia de $T$. evansi que nos discos sem teia (g.l. $=42, \mathrm{t}=2,95, \mathrm{p}<0,05$ e g.l. $=42, \mathrm{t}=2,39, \mathrm{p}<0,05$, respectivamente) (Tabela 1 ).

A teia produzida por T. evansi afetou diferentemente as três espécies de predadores. A predação por C. externa foi negativamente afetada pela presença da teia nos dois últimos instares larvais. É possível que a teia tenha impedido os movimentos desse predador ou dificultado o ataque aos ácaros que estavam em seu interior. Esse fenômeno já foi observado com o percevejo predador, Orius laevigatus (Fieber) (Hemiptera: Anthocoridae), ao predar larvas do tripes Frankliniella occidentalis (Pergande) (Thysanoptera: Thripidae) na presença de teia do ácaro rajado Tetranychus urticae Koch (Acari: Tetranychidae) (Venzon et al., 2000). Apesar da possibilidade de essas espécies ocorrerem nas mesmas plantas, C. externa possivelmente forrageia em locais nos quais não há presença do ácaro, o que evita o contato com a teia. Em plantas de tomate, é comum também a ocorrência de pulgões, que constituem alimento nutricional adequado para essa espécie de predador (Barbosa et al., 2008).

Quanto aos coccinelídeos, as respostas à presença da teia foram variadas. Para E. connexa, somente o último instar larval foi afetado negativamente. A predação foi $23 \%$ inferior em discos com teia em comparação com discos sem teia. É possível que esse predador, nas fases jovens iniciais, explore locais ocupados por T. evansi e os abandone com o passar do tempo. Apesar de não ser um alimento completo para E. connexa (Sarmento et al., 2004), esse ácaro pode representar uma alternativa a ser utilizada em períodos de escassez de suas presas preferenciais (i.e. pulgões).

Em C. sanguinea, as larvas de primeiro e terceiro instares foram afetadas negativamente pela presença da teia. Em presença de teia, as percentagens de predação do primeiro e do terceiro instar foram 46 e $48 \%$ menores, respectivamente. No entanto, no último instar larval, a eficiência desse predador foi $39 \%$ superior em discos com a presença de teia. Essa diferença poderia ser explicada em consequência de o último instar ser mais voraz e maior que os primeiros e, com isso, conseguir sobrepor o efeito negativo da teia. Nos primeiros instares larvais, esse efeito negativo pode ser uma das causas do comportamento das fêmeas de $C$. sanguinea, pois os habitats em que larvas de coccinelídeos ocorrem são determinados pela preferência de oviposição das fêmeas, que evitam as partes da planta ocupadas por T. evansi (Hodek, 1996). Adicionalmente, as fêmeas de C. sanguinea apresentam pouca atração por odores de plantas de tomate ocupadas por esse ácaro e preferem plantas infestadas por pulgões (Sarmento et al., 2007). Pelos resultados deste trabalho, pode-se inferir que, em parte, isso pode ser decorrente da inadequação de tais plantas para o futuro desenvolvimento da prole em razão da presença da teia do ácaro. Além disso, Oliveira et al. (2005) mostraram que larvas de C. sanguinea não completaram seu ciclo biológico quando alimentadas somente com T. evansi, que foram fornecidos sem a retirada da teia produzida pelo ácaro.

A presença da teia de T. evansi pode levar os predadores a abandonar locais da planta cobertos com teia, e a forragear em outras áreas ou mesmo em outras plantas. Furuichi et al. (2005) mostraram que a preferência do ácaro predador Neoseiulus womersleyi (Schicha) (Acari: Phytoseiidae) por uma espécie de presa é determinada pela presença de teia e não pela qualidade do alimento. Ao testar combinações de teia e ovos de duas espécies de ácaros fitófagos, os autores observaram que o ácaro predador prefere o ambiente com a teia mais adequada ao seu forrageamento, independentemente da espécie da presa. A teia também pode ser usada como parâmetro de escolha de plantas 
hospedeiras para oviposição pelas fêmeas adultas desses predadores, que evitam plantas densamente infestadas. Nos experimentos aqui conduzidos, não foi possível avaliar os comportamentos de escape e de evitação, pois os testes foram realizados em arenas confinadas.

A teia do ácaro-vermelho é um importante parâmetro a ser considerado em estudos de seleção de agentes de controle biológico para $T$. evansi. Além de prejudicar a arquitetura da planta hospedeira, ela reduz a taxa fotossintética, facilita a dispersão dos ácaros fitófagos para novas plantas e compromete a ação dos inimigos naturais.

\section{Conclusão}

A teia de Tetranychus evansi pode reduzir a eficiência de predação dos seus inimigos naturais e esse mecanismo de defesa varia em efetividade conforme a espécie de predador e sua fase biológica.

\section{Agradecimentos}

Ao Conselho Nacional de Desenvolvimento Científico e Tecnológico e à Fundação de Amparo à Pesquisa do Estado de Minas Gerais, pelo apoio financeiro ao projeto de pesquisa e pelas bolsas concedidas.

\section{Referências}

BARBOSA, L.R.; CARVALHO, C.F. de; SOUZA, B.; AUAD, A.M. Efficiency of Chrysoperla externa (Hagen, 1861) (Neuroptera: Chrysopidae) in the Myzus persicae (Sulzer, 1776) (Hemiptera: Aphididae) population reduction in sweet pepper (Capsicum annuum L.). Ciência e Agrotecnologia, v.32, p.1113-1119, 2008.

BETTIOL, W.; GHINI, R.; GALVAO, J.A.H.; SILOTO, R.C. Organic and conventional tomato cropping systems. Scientia Agricola, v.61, p.253-259, 2004.

BONATO, O. The effect of temperature on life history parameters of Tetranychus evansi (Acari: Tetranychidae). Experimental and Applied Acarology, v.23, p.11-19, 1999.

CLOUTIER, C.; JOHNSON, S.G. Predation by Orius tristicolor (Hemiptera: Anthocoridae) on Phytoseiulus persimilis (Acarina: Phytoseiidae): testing for compatibility between biological control agents. Environmental Entomology, v.22, p.477-482, 1993.

CRAWLEY, M.J. The R Book. England: Wiley, 2007. 982p.

FERRERO, M.; MORAES, G.J.D.; KREITER, S.; TIXIER, M.S.; KNAPP, M. Life tables of the predatory mite Phytoseiulus longipes feeding on Tetranychus evansi at four temperatures (Acari: Phytoseiidae, Tetranychidae). Experimental \& Applied Acarology, v.41, p.45-53, 2007.

FLECHTMANN, C.H.W. Ácaros de importância agrícola. 5.ed. São Paulo: Nobel, 1983. 189p.

FURTADO, I.P.; MORAES, G.J. de; KREITER, S.; TIXIER, M.S.; KNAPP, M. Potential of a Brazilian population of the predatory mite Phytoseiulus longipes as a biological control agent of Tetranychus evansi (Acari: Phytoseiidae, Tetranychidae). Biological Control, v.42, p.139-147, 2007.

FURUICHI, H.; YANO, S.; TAKAFUJI, A.; OSAKABE, M. Prey preference of the predatory mite Neoseiulus womersleyi Schicha is determined by spider mite webs. Journal of Applied Entomology, V.129, p.336-339, 2005.

GAMARRA, D.C.; BUENO, V.H.P.; MORAES, J.C.; AUAD, A.M. Influence of glandular trichomes of Solanum berthaultii on the predation of Scymnus (Pullus) argentinicus (Weise) (Coleoptera: Coccinellidae) on Myzus persicae (Sulzer) (Homoptera: Aphididae). Anais da Sociedade Entomológica do Brasil, v.27, p.59-65, 1998.

GERSON, U. Webbing. In: HELLE, W; SABELIS, M.W. (Ed.). Spider mites: their biology, natural enemies and control. Amsterdam: Elsevier, 1985. p.223-231.

HODEK, I. Food relationships. In: HODEK, I.; HONEK, A. Ecology of Coccinellidae. Dordrecht: Kluwer Academic, 1996. p.143-238.

KRIPS, O.E.; KLEIJN, P.W.; WILLEMS, P.E.L.; GOLS, G.J.Z.; DICKE, M. Leaf hairs influence searching efficiency and predation rate of the predatory mite Phytoseiulus persimilis. Experimental and Applied Acarology, v.23, p.119-131, 1999.

KUHN, M.; WESTON, S.; WING, J.; FORESTER, J. The contrast package. 2008. Disponível em <http://cran.nedmirror.nl/web/ packages/contrast/vignettes $>$. Acesso em: 07 out. 2009.

MATOS, C.H.C.; PALLINI, A.; CHAVES, F.F.; SCHOEREDER, J.H.; JANSSEN, A. Do domatia mediate mutualistic interactions between coffee plants and predatory mites? Entomologia Experimentalis et Applicata, v.118, p.185-192, 2006.

MICHALSKA, K. Climbing of leaf trichomes by eriophyid mites impedes their location by predators. Journal of Insect Behavior, v.16, p.833-844, 2003.

MORAES, G.J. de; MCMURTRY, J.A. Comparison of Tetranychus evansi and Tetranychus urticae (Acari: Tetranychidae) as prey for eight species of phytoseiid mites. Entomophaga, v.30, p.393-397, 1985.

OLIVEIRA, E.E.; OLIVEIRA, C.L.; SARMENTO, R. de A.; FADINI, M.A.M.; MOREIRA, L.R. Aspectos biológicos do predador Cycloneda sanguinea (Linnaeus, 1763) (Coleoptera: Coccinellidae) alimentado com Tetranychus evansi (Baker e Pritchard, 1960) (Acari: Tetranychidae) e Macrosiphum euphorbiae (Thomas, 1878) (Hemiptera: Aphididae). Bioscience Journal, v.21, p.33-39, 2005.

PALLINI, A.; JANSSEN, A.; SABELIS, M.W. Predators induce interspecific herbivore competition for food in refuge space. Ecology Letters, v.1, p.171-177, 1998. 
PICANÇO, M.C.; BACCI, L.; CRESPO, A.L.B.; MIRANDA, M.M.M.; MARTINS, J.C. Effect of integrated pest management practices on tomato production and conservation of natural enemies. Agricultural and Forest Entomology, v.9, p.327-335, 2007.

RODA, A.; NYROP, J.; DICKE, M.; ENGLISH-LOEB, G. Trichomes and spider mite webbing protect predatory mites eggs from intraguild predation. Oecologia, v.125, p.428-435, 2000.

RODA, A.; NYROP, J.; ENGLISH-LOEB, G.; DICKE, M. Leaf pubescence and two-spotted spider mite webbing influence phytoseiid behavior and population density. Oecologia, v.129, p.551-560, 2001.

ROSA, A.A.; GONDIM, M.G.C.; FIABOE, K.K.M.; MORAES, G.J. de; KNAPP, M. Predatory mites associated with Tetranychus evansi Baker \& Pritchard (Acari: Tetranychidae) on native solanaceous plants of coastal Pernambuco State, Brazil. Neotropical Entomology, v.34, p.689-692, 2005.

SABELIS, M.W.; BAKKER, F.M. How predatory mites cope with the web of their tetranychid prey - a functional view on dorsal chaetotaxy in the Phytoseiidae. Experimental \& Applied Acarology, v.16, p.203-225, 1992.

SABELIS, M.W.; BAKKER, F.M.; BOLLAND, H.R. Hypotrichy in the Phytoseiidae - adaptation or phylogenetic constraint? Experimental \& Applied Acarology, v.18, p.241-245, 1994.

SAITO, Y. Life types of spider mites. In: HELLE, W.; SABELIS, M.W. (Ed.). Spider mites: their biology, natural enemies and control. Amsterdam: Elsevier, 1985. p.253-264.
SARMENTO, R.A.; OLIVEIRA, H.G. de; HOLTZ, A.M.; SILVA, S.M.; SERRÃO, J.E.; PALLINI, A. Fat body morphology of Eriopis connexa (Coleoptera, Coccinelidae) in function of two alimentary sources. Brazilian Archives of Biology and Technology, v.47, p.407-411, 2004.

SARMENTO, R.A.; VENZON, M.; PALLINI, A.; OLIVEIRA, E.E.; JANSSEN, A. Use of odours by Cycloneda sanguinea to asses patch quality. Entomologia Experimentalis et Applicata, v.124, p.313-318, 2007.

TOSCANO, L.C.; AUAD, A.M.; FIGUEIRA, L.K. Comportamento de Chrysoperla externa (Hagen, 1861) em genótipos de tomateiro infestados com ovos de Bemisia tabaci (Gennadius, 1889) biótipo B em laboratório. Arquivos do Instituto Biológico, v.70, p.117-121, 2003.

VANTORNHOUT, I.; MINNAERT, H.L.; TIRRY, L.; DE CLERCQ, P. Influence of diet on life Table parameters of Iphiseius degenerans (Acari: Phytoseiidae). Experimental and Applied Acarology, v.35, p.183-195, 2005.

VENZON, M.; JASSEN, A.; PALLINI, A.; SABELIS, M.W. Diet of a polyphagous predator affects refuge seeking of its thrips prey. Animal Behaviour, v.60, p.369-375, 2000.

WEKESA, V.W.; MANIANIA, N.K.; KNAPP, M.; BOGA, H.I. Pathogenicity of Beauveria bassiana and Metarhizium anisopliae to the tobacco spider mite Tetranychus evansi. Experimental and Applied Acarology, v.36, p.41-50, 2005.

Recebido em 27 de fevereiro de 2009 e aprovado em 23 de agosto de 2009 